\title{
Quadruple context-free L-System mathematical tools as origin of biologicalevolution
}

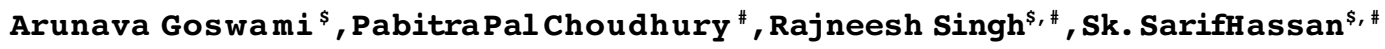 \\ \$BiologicalSciences Division and "Applied StatisticsUnit, Indian Statisticalinstitute,203, B. T. \\ Road, Kolkata 700108 , India
}

\begin{abstract}
Itiswellknown that A, T, G, C annealed together early in evolution and the long stretch of DNA was found which ultimately resulted into chromosomes of different organisms. But it is unclear tilldate how exons, introns, conserved protein domains was formed. Using the DNA sequences of the largestknown gene-familypresent in human genome, i.e.,olfactory receptors and simplest possible quadruple context-free L-Systems, we show that conserved protein domains and intergenic regions which liesat the heart of the biologicalevolution started with a sixteen base-pairs stretch of DNA.
\end{abstract}

\section{Use of quadruple context-free L-Systems on olfactory receptors (OR)}

ORs constitute the largest known multigene family involved in sense of smell of different organisms. There are $\sim 388$ functional and $\sim 414$ pseudo OR genes are present in human genome [1]. Researchers have found that except two chromosomes, oRs are unevenly distributed in 21 chromosomes. OR 1 familycontains 28 fulllengths and 5 pseudo ORs as per HORDE database (https://senselab.med.yale.edu/ORDB/files/humanorseqanal.html). Fig. 1 shows the Clustalw (http://align.genome.jp/) data of extreme 5'end stretch of 29 fulllength ORs.

Fig. 1

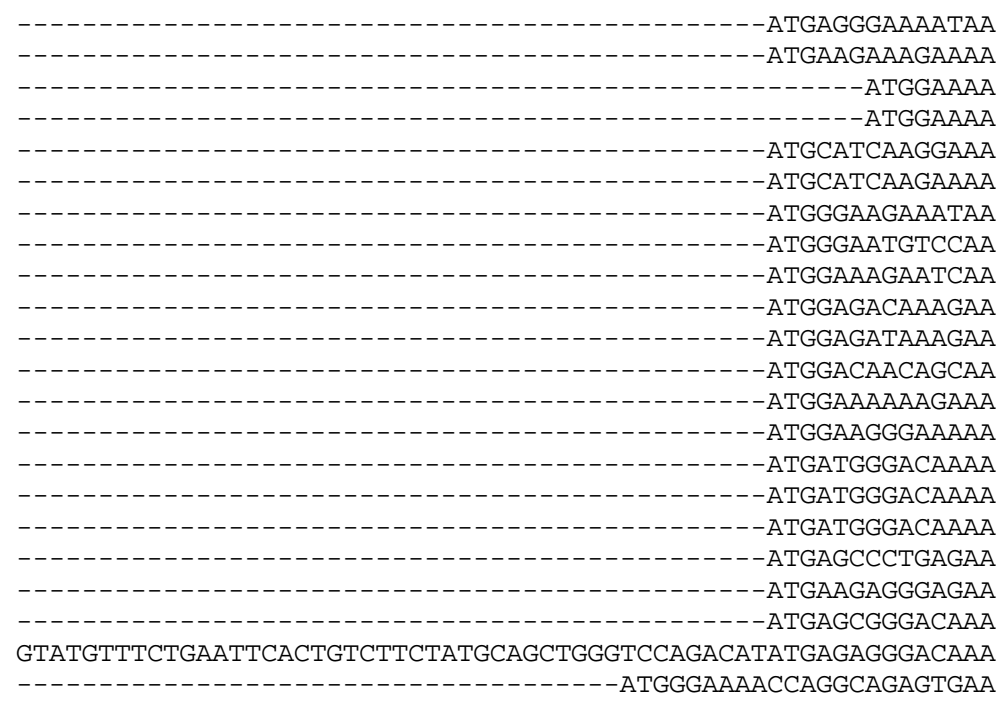




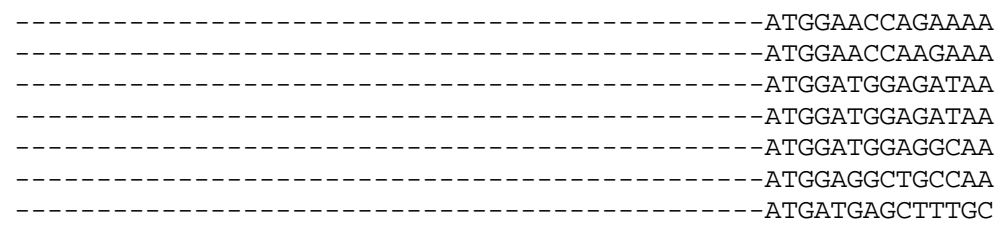

CC--AGTCCTCTA---CACTGGAATTCATC-CTCCTGGGAGTTACTGGTCAGCAGGAACA TC--AATCCTTTA---ACCTGGATTTTATT-CTCCTGGGAGTTACTAGTCAGCAAGAACA CC--AATCCAGCA---TTTCTGAATTTTTC-CTCCGAGGAATATCAGCGCCTCCAGAGCA CC--AATCCAGCA---TTTCTGAATTTTTC-CTCCGAGGAATATCAGCGCCTCCAGAGCA CC--AAACCACCA---TCACTGAATTCATT-CTCCTGGGATTTTTCAAGCAGGATGAGCA CC--AAACCACCA---TCACTGAATTCATT-CTCCTGGGACTCTCCAACCAGGCTGAACA CC--TAACAAGAC---CCTCTGAATTCATC-CTCCTTGGACTCTCCTCTCGACCTGAGGA CC--TGACAAGAC---TCTCTGAATTTATT-CTCTTGGGACTCTCCTCTCGGTCTGAAGA CC--ACACCAGCAGTGTCTCCGAGTTTATC-CTCCTGGGACTCTCCTCCCGGCCTGAGGA TT--ATAGCAGCAGCACCTCAGGCTTCATC-CTCCTGGGCCTCTCTTCCAACCCTAAGCT CT--ACAGCAGCAGCACCTCAGGCTTCATC-CTCCTGGGCCTCTCTTCCAACCCTCAGCT CT--GGACCAGTG---TGTCCCATTTTGTT-CTCTTGGGCATTTCCACCCACCCAGAAGA TC--TAACAGTTG---TCAGGGAATTCGTC-CTTCTGGGACTTCCTAGCTCAGCAGAGCA TC--AAACCAATA---TCTCTGAATTTCTC-CTCCTGGGCTTCTCAAGTTGGCAACAACA TC--AAACCAGCA---TCTCAGACTTCCTG-CTCCTGGGCCTGCCCATCCAACCAGAGCA TC--AAACCAGCA---TCTCAGACTTCCTG-CTCCTGGGCCTGCCCATCCAACCAGAGCA TC--AAACCAGCA---TCTCAGACTTCCTG-CTCCTGGGCCTGCCCATCCAACCAGAGCA CC--AGAGCAGCG---TGTCCGAGTTCCTC-CTTCTGGGCCTCCCCATCCGGCCAGAGCA TC--AGAGCAGTG---TGTCTGAGTTCCTC-CTCCTGGACCTCCCCATCTGGCCAGAGCA CC--AGTCGAGTG---TCTCCGAGTTCCTC-CTCCTGGGACTCTCCAGGCAGCCCCAGCA CC--AGT-GAGTG---TCTCCGAGTTCCTC-CTCTTGGGACTCTCCAGGCAGCCCCAGCA CC--AAACCACTG---TTTCAGACTTCCTC-CTTCTAGGACTCTCTGAGTGGCCAGAGGA GC--AAACCGAAA---TCTCAGAATTCTTC-CTCCAGGGACTCTCAGAAAAGCCAGAGCA CC--AAACCAGTG---CATCTCAATTCATC-CTCCTGGGACTCTCAGAAAAGCCAGAGCA CC--AGAGTGAGA---ACTCACAGTTCCTT-CTCCTGGGGATCTCAGAGAGTCCTGAGCA CC--AGAGTGAGA---ACTCACAGTTCCTT-CTCCTGGGGATCTCAGAGAGTCCTGAGCA CC--AGAGTGAAG---GTTCAGAGTTCCTT-CTCCTGGGGATGTCAGAGAGTCCTGAGCA TG--AGTCTTCAGAGGGAATCTCATTCGTT-TTATTGGGACTGACAACAAGTCCTGGACA CCCTAATGCTTCACACTCTCCGGTTTTTTTGCTCCTTGGGTTCTCGAGAGCTAACATCTC

OR1B1

Fig. 1 Legend. ClustalW analysisof 29 (OR1E1 and OR1E6 are same) OR 1 familymembers of OR present in HORDE. Asterisks indicate conserved nucleotides.

Fig. 1 shows that out of aforesaid 28,23 ORs has distinct (each with a set of 4 bp) 16 bp sequence at the extreme 5'end which could be used to design production rules of L-Systems [2].Fig. 2 shows three different classes of L-Systems, which have been derived from 28 oRs of family 1 .

First class, designated as 'Class- 1 ' consists of 19 following OR1 family sequences- OR1A1, OR1B1, OR1E1 (same as OR1E6), OR1E2, OR1F1, OR1F12, OR1I1, OR1J2, OR1J4, OR1K1, OR1L1, OR1L3, OR1L4, OR1L6, OR1L8, OR1N2, OR1Q1, OR1S1, OR1S2. For these sequences, when context free L-System production rules were made from the extreme 5'end of the DNA sequences, with axiom: A, the rules were found to non-overlapping. For example, for OR $1 A 1$ the production rules are A ATGA, C GGGA, T AAAT, G AACC. One of the observations, in this class is OR1S1, OR1S2. Although their first sixteen base-pair sequence same (i.e., their production rules are same), OR1S1 and OR1S2 has different DNA sequences in theirexons. Using context-free L-Systems over a number of iterations one can introduce mutations in terms 
of insertions to form OR1S 1 to OR1S2 and vice-versa $[3,4,5,6]$. As context-free L-System isan irreversiblesystem so when OR1S1/ OR1S2 willbe made from OR1S2/OR1S1 after the exonic sequence a large intergenic sequence would be generated. OR1S1 and OR 1S2 are present in chromosome 11. Although no subfamilymembers of OR1S are present in the chromosome 11 , itmay be that from a large number of other ORs present in the same chromosome ( 390 ORs in HORDE) have given rise to OR1S1/OR1S2. From this study it isclear that after OR1S1 and OR1S2, itisdifficultto generate same subfamilymembers and a large intergenic region would be produced afterthe generation of OR1S1/OR1S2.

Second class, designated as 'Class-1I'Consistsof three following OR1 family sequences-OR1C1, OR1N1, OR1N3. The production rules for this class has a unique feature that one of the production rule gives poly A sequences. For example, for OR $1 \mathrm{C} 1$ the production rules are A ATGG, C AAAA, T AAGA, G AATC.

Third class, designated as 'Class-1II'Consists of five following OR 1 family sequences- OR $1 A 2$, OR1D2, OR1D4, OR1D5, OR1M1. Unique feature of class-IIIis that two of the production rules are similar. For example, for OR1A2 the production rules are A ATGA, C AGAA, T AGAA, G AATC. As the production rule for $\mathrm{T}$ and $\mathrm{C}$ are same, so successive iterations willincrease the amount of repetitive DNA sequence generated by the class-III production rules. OR1D subfamily has a singlepseudogene OR1D3P which ismost similarto OR1D2. As described above OR1D3P was produced from OR1D2. We took OR1D3P sequence and italso fallin the class-III category of ORs.

Therefore from the above discussion itisclearthis isa new classification system based on only first1 $6 \mathrm{bp}$ sequence of ORs and not on the sequence homology of either DNA or protein of the entireor sequence.

\section{Evolutionary implications}

Let us assume that $16 \mathrm{bp}$ sequence of alloRs (Class-I,Class-IIand Class-III)of OR family 1 were present as individual sequences somewhere in the evolutionary scale. This is not an assumption per se as they are present in all the subfamilymembers of the largestmultigene family known in the genome i.e.,oR which ispresent in the human genome tilldate. Each of these $16 \mathrm{bp}$ sequences willgive rise to evolution of unique protein domains (supplementary data provided).For example we took OR1L subfamilymembers which have 5 me mbers (OR1L1, OR1L3, OR1L4, OR1L6 and OR1L8). Applying the same method as described in Ref. 6 (and supplementary information provided herewith) we generated following conserved protein domains.OR1L1 gave $80 \%-200 \%$ alignment score with a hypotheticalprotein from Giant Panda. SimilarlyoR1L3 gave a composite conserved protein domain from Californiapurple sea urchin with homology ranging from 40\%-200\%. Using the similar methodology OR1L 4 and OR1L6 derived L-Systems could not produce any ORFs and therefore consequently no conserved 
protein domains were formed. We conjecture that these two aforesaid and following LSystems (Axiom: A) A ATGG, C AGAC, TAAAG, G AATT and A ATGG, C AGAT, T AAAG, G AACT have not been so far for producing conserved protein domains but may have been used formaking intergenic DNA sequence. OR1L8 when was used in the same model was found to pick up suprabasin protein from three differentorganisms, viz.Dog, Horse and Giant Panda.

Therefore one can visualize very very early in evolution if nature could derive any of the aforesaid three classes (Class-I,class-II,class-III)L-Systems which means generation of a simple stretch of $16 \mathrm{bp}$ DNA sequence then extension of the DNA chain using DNA polymerase can happen. We showed in this paper that extension of this kind of L-System can lead to meaningfulconserved protein domains as wellas intergenicDNA sequences. To the best of our knowledge, this is the firstreport which showed how evolution started with a 16 bp DNA sequences.

ACKNOWLEDGMENTS: This work was supported by the Department of Biotechnology (DBT), New Delhi,grants (BT/PR9050/NNT/28/21/2007 and BT/PR8931/NNT/28/07/2007 to AG) and NAIP-ICAR-World Bank grant (Comp-4/C3004/2008-09; Project leader:AG) and ISIplan projects for 2001-2011 to A. G. Authors would like to thank visiting students, Ranita Guha and Shantanav Chakrovorty for theirvaluable inputs in computations.

\section{References}

1. Malnic B, Godfrey P-A and Buck L-B (2004) The human olfactory receptor gene family; Proc. Natl.Acad. Sci.USA 101 2584-2589 Erratum in: Proc. Natl.Acad. Sci.USA 2004 1017205

2. Prusinkiewicz, P. and Lindenmayer, A. (1990) in the algorithmic beauty of plants (New York: Springer-Verlag).

3. Hassan, S. Sk., Choudhury, P. P., Pal, A., Brahmachary, R. L. and Goswami, A . (2010) LSystems: A Mathematical Paradigm for Designing Full Length Genes and Genomes. Global Journal of Computer Science and Technology, 10: 119-122, category: I.2.1, J.3, and G.1.0. (Published in June, 2010)

4. Hassan, S. Sk., Choudhury, P. P., Pal, A., Brahmachary, R. L. and Goswami. A. (2010) Designing exons for human olfactory receptor gene subfamilies using a mathematical paradigm. Journal of Biosciences, volume 35, number 3: 389-393.

5. Hassan, S. Sk., Choudhury, P. P., Pal, A., Brahmachary, R. L. and Goswami, A. (2010) Combination of L-systems: For Designing Human Olfactory Receptor Pseudo-gene, OR 1 D 3 P. International Journal of Computational Cognition, (Publisher: Yang's Scientific Research Institute,USA) (Accepted for publication in 2011).

6. Goswami, Arunava, Singh, Rajneesh, Choudhury, Pabitra, and Hassan, Sk. SarifHassan. Designing L-Systems for making three and sixopen reading frames from the leading strand of a single DNA molecule. Available from Nature Precedings <http://hdl.handle.net/10101/npre.2010.4844.1>(2010). 


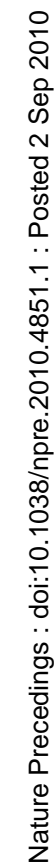

